This is the final peer-reviewed accepted manuscript of:

Bonfiglioli A. (2019) Potential Theory Results for a Class of PDOs Admitting a Global Fundamental Solution. In: Delgado J., Ruzhansky M. (eds) Analysis and Partial Differential Equations: Perspectives from Developing Countries. Springer Proceedings in Mathematics \& Statistics, vol 275. Springer, Cham

The final published version is available online at: https://doi.org/10.1007/978-3-030-05657$\underline{56}$

Rights / License:

The terms and conditions for the reuse of this version of the manuscript are specified in the publishing policy. For all terms of use and more information see the publisher's website.

This item was downloaded from IRIS Università di Bologna (https://cris.unibo.it/)

When citing, please refer to the published version. 


\title{
Potential Theory results for a class of PDOs admitting a global fundamental solution
}

Andrea Bonfiglioli

\begin{abstract}
We outline several results of Potential Theory for a class of linear partial differential operators $\mathscr{L}$ of the second order in divergence form. Under essentially the sole assumption of hypoellipticity, we present a non-invariant homogeneous Harnack inequality for $\mathscr{L}$; under different geometrical assumptions on $\mathscr{L}$ (mainly, under global doubling/Poincaré assumptions), it is described how to obtain an invariant, non-homogeneous Harnack inequality. When $\mathscr{L}$ is equipped with a global fundamental solution $\Gamma$, further Potential Theory results are available (such as the Strong Maximum Principle). We present some assumptions on $\mathscr{L}$ ensuring that such a $\Gamma$ exists.
\end{abstract}

\section{Introduction}

The keystones of classic Potential Theory were established in the century elapsing between the 20's of the nineteenth century and the 20's of the twentieth century. During this period, the following topics were studied, regarding the classic Laplace operator $\Delta$ :

- 1823: the Dirichlet problem (Poisson);

- 1828: the Green's function (Green);

- 1839: the Maximum Principle (Earnshaw);

- 1840: mean value properties (Gauss);

- 1870: further results on the Dirichlet problem (Schwarz);

- 1887: convergence properties (Harnack);

- 1890: barrier functions (Poincaré);

- 1923 ca: weak solvability (Perron, Wiener, Brelot);

Andrea Bonfiglioli

Dipartimento di Matematica, Università degli Studi di Bologna, Piazza di Porta San Donato, 5, 40126 Bologna, Italy, e-mail: andrea.bonfiglioli6@unibo.it 
- 1930: representation theory and the potential function (Riesz);

- 1929: axiomatic Potential Theory (Kellogg).

All these topics are deeply interrelated, and each of them could hardly exist without the others. However, if we were to select a restricted number of them according to their most capital role in Potential Theory, we would point out the undisputable importance of the Green function, the Maximum Principle, and the convergence properties. In this paper we outline some recent (or very recent) results concerning the latter topics, for classes of linear partial differential operators (PDOs, in the sequel) of the second order in divergence form.

As an outline of the paper, the exposition is thusly presented:

1. in Section 2, we introduce a rather general class of hypoelliptic PDOs $\mathscr{L}$, and we show how to obtain a non-invariant homogenous Harnack inequality for $\mathscr{L}$; by restricting the class considered, we then describe how to prove an invariant and non-homogenous inequality (Section 4);

2. when $\mathscr{L}$ possesses a well-behaved global Green function $\Gamma$ (also called fundamental solution), more Potential Theoretic results are available, including Maximum Principles (Section 3);

3 . due to the importance of $\Gamma$, under stronger assumptions on $\mathscr{L}$, we provide sufficient conditions for its existence (Section 5).

\section{A non-invariant Harnack inequality}

On Euclidean space $\mathbb{R}^{N}$, we consider a PDO $\mathscr{L}$ of the second order in the following divergence form ("weighted" by a density $V$ )

$$
\mathscr{L}:=\frac{1}{V(x)} \sum_{i, j=1}^{N} \frac{\partial}{\partial x_{i}}\left(V(x) a_{i, j}(x) \frac{\partial}{\partial x_{j}}\right),
$$

where the functions $V$ and $a_{i, j}$ fulfil the following assumptions:

- $V$ and any $a_{i, j}$ are $C^{\infty}$ from $\mathbb{R}^{N}$ to $\mathbb{R}$;

- $V(x)>0, A(x):=\left(a_{i, j}(x)\right)_{i, j}$ is symmetric and positive semi-definite at any $x \in$ $\mathbb{R}^{N}$

- assumption (NTD): $\mathscr{L}$ is non-totally-degenerate at any point, i.e., $A(x) \neq 0$ for any $x \in \mathbb{R}^{N}$;

- assumption (HY): $\mathscr{L}$ is $C^{\infty}$-hypoelliptic on every open subset of $\mathbb{R}^{N}$.

There are examples of operators satisfying our assumptions in: Lie group theory (e.g., sub-Laplacian operators [26]); CR Geometry and Riemannian Geometry; several complex variables; Brownian motion; Stochastic PDEs.

Remark 1. We observe that there are examples of operators (with infinitely-degenerate coefficients) fulfilling all of our assumptions but outside the well-studied class of Hörmander operators; for example: 


$$
\begin{gathered}
\frac{\partial^{2}}{\partial x_{1}^{2}}+\left(\exp \left(-\frac{1}{x_{1}^{2}}\right) \frac{\partial}{\partial x_{2}}\right)^{2} \text { in } \mathbb{R}^{2}, \\
\frac{\partial^{2}}{\partial x_{1}^{2}}+\left(\exp \left(-\frac{1}{\sqrt{\left|x_{1}\right|}}\right) \frac{\partial}{\partial x_{2}}\right)^{2}+\frac{\partial^{2}}{\partial x_{3}^{2}} \quad \text { in } \mathbb{R}^{3}, \\
\frac{\partial^{2}}{\partial x_{1}^{2}}+\left(\exp \left(-1 /\left|x_{1}\right|\right) \frac{\partial}{\partial x_{2}}\right)^{2}+\left(\exp \left(-1 /\left|x_{1}\right|\right) \frac{\partial}{\partial x_{3}}\right)^{2} \text { in } \mathbb{R}^{3}, \\
\frac{\partial^{2}}{\partial x_{2}^{2}}+\left(x_{2} \frac{\partial}{\partial x_{1}}\right)^{2}+\frac{\partial^{2}}{\partial x_{4}^{2}}+\left(\exp \left(-1 / \sqrt[3]{\left|x_{1}\right|}\right) \frac{\partial}{\partial x_{3}}\right)^{2} \text { in } \mathbb{R}^{4},
\end{gathered}
$$

respectively studied by Fediǔ [41]; Kusuoka, Stroock [63]; Christ [34]; Morimoto [65], who proved that the above PDOs are indeed $C^{\infty}$-hypoelliptic.

To specify the role of $V$, if $\mathrm{d} x$ is Lebesgue measure in $\mathbb{R}^{N}$, in the sequel we set

$$
\mathrm{d} \mu(x):=V(x) \mathrm{d} x .
$$

Clearly, $\mathscr{L}$ is formally self-adjoint in $L^{2}\left(\mathbb{R}^{N}, \mathrm{~d} \mu\right)$ on the smooth compactly-supported functions:

$$
\int \varphi \mathscr{L} \psi \mathrm{d} \mu=\int \psi \mathscr{L} \varphi \mathrm{d} \mu, \quad \forall \varphi, \psi \in C_{0}^{\infty}\left(\mathbb{R}^{N}\right)
$$

The opportunity to permit $V$ to be non-identically 1 comes from our interest in second-order operators coming from Lie group theory; indeed, one can find in [6, Example 1.1] some examples of operators of the form (1), where $V \neq 1$ is the density of the Haar measure $\mu$ of a Lie group $G$ whose underlying manifold is $\mathbb{R}^{N}$, and $X_{1}, \ldots, X_{m}$ is a family of generators of the Lie algebra of $G$. In this case, it appears very natural to consider sub-Laplace operators of the form

$$
\mathscr{L}=-\sum_{j=1}^{m} X_{j}^{* \mu} X_{j},
$$

where $X_{j}^{* \mu}$ denotes the adjoint of the vector field $X_{j}$ in $L^{2}(G, \mathrm{~d} \mu)$. Such an $\mathscr{L}$ can always be written in the form (1); it satisfies (HY) in force of Hörmander's hypoellipticity theorem (since $X_{1}, \ldots, X_{m}$ are Lie-generators of $\operatorname{Lie}(G)$ ).

For another explicit example, see the next one:

Example 1. Consider the Lie group $G=\left(\mathbb{R}^{3}, *\right)$ where

$$
\left(x_{1}, x_{2}, x_{3}\right) *\left(y_{1}, y_{2}, y_{3}\right)=\left(x_{1}+y_{1}, x_{2}+y_{2}, x_{3}+y_{3} e^{x_{1}}\right) ;
$$

it is natural to consider the Haar measure $\mu$ on $G$ defined by $\mu=V(x) \mathrm{d} x$, with $V(x)=e^{-x_{1}}$; if we also consider the Lie generators $X_{1}, X_{2}$ of Lie $(G)$ given by $X_{1}=$ $\partial_{x_{1}}$ and $X_{2}=\partial_{x_{2}}+e^{x_{1}} \partial_{x_{3}}$, then the operator $\mathscr{L}=-X_{1}^{* \mu} X_{1}-X_{2}^{* \mu} X_{2}$ has the form

$$
\mathscr{L}=\left(\partial_{x_{1}}\right)^{2}+\left(\partial_{x_{2}}\right)^{2}+e^{2 x_{1}}\left(\partial_{x_{3}}\right)^{2}+2 e^{x_{1}} \partial_{x_{2}, x_{3}}-\partial_{x_{1}} .
$$

$\mathscr{L}$ enjoys the form (1) with the above $V(x)$ and with 


$$
A(x)=\left(\begin{array}{ccc}
1 & 0 & 0 \\
0 & 1 & e^{x_{1}} \\
0 & e^{x_{1}} & e^{2 x_{1}}
\end{array}\right)
$$

but it cannot be written as a pure divergence form operator $\operatorname{div}(B(x) \nabla)$, whence the opportunity to consider operators of the more general form (1). $\mathscr{L}$ also enjoys assumptions (NTD) and (HY) (the latter is due to Hörmander's hypoellipticity theorem, as already remarked).

Apart from the elliptic case, in the existing literature, as regards the Maximum Principles and the Harnack inequality for (divergence-form) degenerate-elliptic PDOs under a subelliptic assumption (or assuming that the degeneracy be controlled by some Muckenhoupt weights), there are several references starting from the 1980's: Chanillo and Wheeden [33]; Fabes, Jerison and Kenig [37]; Fabes, Kenig and Serapioni [38]; Fefferman and Phong [40]; Franchi and Lanconelli [45]; Gutiérrez [52] Jerison and Sánchez-Calle [58].

It is also well known that a natural framework for the Harnack inequality is that of doubling metric spaces (where a doubling condition and a Poincaré inequality are available): Aimar, Forzani and Toledano [3]; Barlow and Bass [5]; Di Fazio, Gutiérrez and Lanconelli [36]; Grigor'yan and Saloff-Coste [51]; Gutiérrez and Lanconelli [53]; Hebisch and Saloff-Coste [54]; Indratno, Maldonado and Silwal [57]; Kinnunen, Marola, Miranda and Paronetto [59]; Mohammed [64]; Saloff-Coste [70].

Without making any subellipticity or Muckenhoupt assumption, nor requiring any underlying doubling/Poincaré metric space setting, in [6] we proved the following Strong Maximum Principle, a key step for the Harnack inequality:

Theorem 1 (Strong Maximum Principle for $\mathscr{L}$ ). Suppose that $\mathscr{L}$ is an operator of the form (1), with $C^{\infty}$ coefficients $V>0$ and $\left(a_{i, j}\right)_{i, j} \geq 0$, and that it satisfies (NTD) and (HY). Let $O \subseteq \mathbb{R}^{N}$ be a connected open set. Then, the following facts hold.

1. Any function $u \in C^{2}(O, \mathbb{R})$ satisfying $\mathscr{L} u \geq 0$ on $O$ and attaining a maximum in $O$ is constant throughout $O$.

2. If $c \in C^{\infty}\left(\mathbb{R}^{N}, \mathbb{R}\right)$ is nonnegative on $\mathbb{R}^{N}$, and if we set

$$
\mathscr{L}_{c}:=\mathscr{L}-c,
$$

then any function $u \in C^{2}(O, \mathbb{R})$ satisfying $\mathscr{L}_{c} u \geq 0$ on $O$ and attaining a nonnegative maximum in $O$ is constant throughout $O$.

We proved Theorem 1 following the approach by Bony in his celebrated 1969 paper [27] concerning with Hörmander sums of squares. However, in view of Remark 1, since our assumptions are general enough to comprise non-Hörmander PDOs we cannot simply recover Bony's argument, which is based on the well-known connectivity theorem of Carathéodory, Chow, Hermann and Rashevskir.

Luckily for us, a theorem by Amano [4] on a connectivity/controllability property for hypoelliptic PDOs allows us to recover Bony's ideas in their full strength 
(thus circumventing the issue caused by non-Hörmander PDOs). Our argument for Theorem 1 is essentially as follows. We set

$$
A_{i}:=\sum_{j=1}^{N} a_{i, j} \frac{\partial}{\partial x_{j}} \quad(i=1, \ldots, N), \quad A_{0}:=\frac{1}{V} \sum_{i=1}^{N} \frac{\partial V}{\partial x_{i}} A_{i}
$$

then we argue in this way (for the details and the nomenclature, see [6]):

1. $A_{1}, \ldots, A_{N}$ are tangent to the set $F(u):=\left\{x \in \Omega: u(x)=\max _{\Omega} u\right\}$, provided $\mathscr{L} u \geq 0$ (Hopf-type lemma);

2. $A_{0}$ is also tangent to $F(u)$ (it is a smooth combination of $A_{1}, \ldots, A_{N}$ );

3 . the tangentiality of $A_{0}, A_{1} \ldots, A_{N}$ ensures the propagation of $F(u)$ along the integral curves of any linear combination of $A_{0}, A_{1}, \ldots, A_{N}$ (Bony);

4. the $C^{\infty}$-hypoellipticity of $\mathscr{L}$ ensures the controllability (hence, a suitable connectivity property) of the set $\operatorname{span}\left\{A_{0}, A_{1}, \ldots, A_{N}\right\}$ (this is the point where we invoke Amano's crucial result).

These facts plainly prove that $F(u)$ is forced to coincide with $\Omega$, as soon as $F(u)$ is non-void. As for (4), we review Amano's geometric result on hypoellipticity: once one has rewritten $\mathscr{L}$ in the following way

$$
\mathscr{L}=\sum_{i=1}^{N} \frac{\partial}{\partial x_{i}}\left(A_{i}\right)+A_{0}
$$

then assumptions (NTD) and (HY) implies a connectivity property of any pair of points of the underlying domain $\Omega$, along the linear combinations of $A_{0}, A_{1}, \ldots, A_{N}$. See [4] for all the details. In order to prove our non-invariant Harnack inequality, we need a further assumption:

- assumption $(\mathbf{H Y})_{\varepsilon}$ : for every $\varepsilon>0, \mathscr{L}-\varepsilon$ is $C^{\infty}$-hypoelliptic on every open set in $\mathbb{R}^{N}$.

The problem of establishing, in general, whether or not our previous assumption $(\mathrm{HY})$ implies $(\mathrm{HY})_{\varepsilon}$ is non-trivial; it appears that having some quantitative information on the loss of derivatives may help in facing this question (personal communication by Parmeggiani). Concerning this issue it is known that, for example, in the complex coefficient case the presence of a zero-order term (even a small $\varepsilon$ ) may drastically alter hypoellipticity (see Stein, [71]; see also the very recent paper [66] by Parmeggiani for related topics).

The role of $(\mathrm{HY})_{\varepsilon}$ is crucial in establishing a comparison argument (analogous to that in [27, Proposition 7.1, p.298]) giving the lower bound

$$
u\left(x_{0}\right) \geq \varepsilon \int_{\Omega} u(y) G_{\mathcal{\varepsilon}}\left(x_{0}, y\right) V(y) \mathrm{d} y \quad \forall x_{0} \in \Omega,
$$

for every nonnegative $\mathscr{L}$-harmonic function $u$ on the open set $\Omega$ which possesses a Green kernel $G_{\varepsilon}(x, y)$ relative to the perturbed operator $\mathscr{L}-\varepsilon$. 
With the aid of (4), with the use of the Green functions related to $\mathscr{L}$ and $\mathscr{L}_{\varepsilon}$, and by means of Theorem 1, in [6] we proved the following Harnack inequality:

Theorem 2 (Harnack Inequality). Suppose that $\mathscr{L}$ is an operator of the form (1), with $C^{\infty}$ coefficients $V>0$ and $\left(a_{i, j}\right) \geq 0$, and suppose it satisfies hypotheses (NTD), $(\mathrm{HY})$ and $(\mathrm{HY})_{\varepsilon}$.

Then, for every connected open set $O \subseteq \mathbb{R}^{N}$ and every compact subset $K$ of $O$, there exists a constant $M=M(\mathscr{L}, O, K) \geq 1$ such that

$$
\sup _{K} u \leq M \inf _{K} u
$$

for every nonnegative $\mathscr{L}$-harmonic function $u$ in $O$. If $\mathscr{L}$ is subelliptic or if it has $C^{\omega}$ coefficients, then assumption $(\mathrm{HY})_{\varepsilon}$ can be dropped.

We remark that the operators in Remark 1 are not subelliptic (nor $C^{\omega}$ ), yet they satisfy hypotheses (NTD), (HY) and $(\mathrm{HY})_{\mathcal{\varepsilon}}$. The lack of subellipticity is a consequence of the characterization of the subelliptic PDOs due to Fefferman and Phong [39, 40] (see also [62, Prop.1.3] or [58, Th.2.1 and Prop.2.1]; the validity of $(\mathrm{HY})_{\varepsilon}$ derives from a result by Kohn, [62]. Thus our Theorem 2 is not a consequence of Bony's results in [27], nor of the results on Harnack inequalities for subelliptic PDOs cited after Example 1.

Technically, we obtained Theorem 2 from the following result (due to Mokobodzki and Brelot, [30, Chapter I]):

Lemma 1. Let L be a second order linear PDO in $\mathbb{R}^{N}$ with smooth coefficients. Suppose the following conditions are satisfied.

(Regularity): There exists a basis $\mathscr{B}$ for the Euclidean topology of $\mathbb{R}^{N}$ (consisting of bounded open sets) such that, for every $\Omega \in \mathscr{B} \backslash\{\varnothing\}$ and for every $\varphi \in C(\partial \Omega, \mathbb{R})$, there exists a unique L-harmonic function $H_{\varphi}^{\Omega} \in C^{2}(\Omega) \cap C(\bar{\Omega})$ solving the Dirichlet problem

$$
\left\{\begin{array}{l}
L u=0 \text { in } \Omega \\
u=\varphi \text { on } \partial \Omega
\end{array}\right.
$$

and satisfying $H_{\varphi}^{\Omega} \geq 0$ whenever $\varphi \geq 0$.

(Weak Harnack Inequality): For every connected open set $O \subseteq \mathbb{R}^{N}$, every compact subset $K$ of $O$ and every $y_{0} \in O$, there exists a constant $C\left(y_{0}\right)=C\left(L, O, K, y_{0}\right)>0$ such that

$$
\sup _{K} u \leq C\left(y_{0}\right) u\left(y_{0}\right),
$$

for every nonnegative L-harmonic function u in $O$.

Then, the thesis of Theorem 2 holds for $L$.

When one takes $L=\mathscr{L}$, the validity of condition '(Regularity)' can be obtained as a consequence of the hypoellipticity of $\mathscr{L}$ (as in [27]); besides, the validity of condition '(Weak Harnack Inequality)' is a consequence of (4) plus some basic Functional Analysis. 
Introducing the topics of the following section, we underline that the Harnack inequality (5) implies the following Brelot convergence property: every increasing sequence of $\mathscr{L}$-harmonic functions (on a connected open set) either converges to $\equiv \infty$ or to an $\mathscr{L}$-harmonic function. This is one of the most crucial axiom (and often one of the most difficult to prove) of the Axiomatic Potential Theory for $\mathscr{L}$ (see [26, Chapter 6]).

\section{Further Potential Theory results}

A most convenient assumption for potential-theoretic problems for operators $\mathscr{L}$ as in (1) is that $\mathscr{L}$ possesses a smooth, global and positive fundamental solution: see the series of papers $[1,7,24,25]$. For the use of the fundamental solution in obtaining the Harnack inequality for Hörmander sums of squares, see: Citti, Garofalo and Lanconelli [35]; Garofalo and Lanconelli [48, 49]; Pascucci and Polidoro [67, 68]; Bramanti, Brandolini, Lanconelli and Uguzzoni [29].

First of all we clarify what we mean by a fundamental solution: for the (formally) self-adjoint operator $\mathscr{L}$ as in (1), we assume the existence of a (global) fundamental solution, that is a function

$$
\Gamma:\left\{(x, y) \in \mathbb{R}^{N} \times \mathbb{R}^{N}: x \neq y\right\} \longrightarrow \mathbb{R} \quad \text { such that: }
$$

1. for every fixed $x \in \mathbb{R}^{N}$ (the so-called 'pole'), $\Gamma_{x}:=\Gamma(x, \cdot)$ belongs to $L_{\mathrm{loc}}^{1}\left(\mathbb{R}^{N}\right)$ and it solves $-\mathscr{L} \Gamma_{x}=\operatorname{Dirac}_{x}$, i.e.,

$$
\int_{\mathbb{R}^{N}} \Gamma(x, y) \mathscr{L} \varphi(y) \mathrm{d} \mu(y)=-\varphi(x), \quad \forall \varphi \in C_{0}^{\infty}\left(\mathbb{R}^{N}\right) .
$$

2. for every fixed $x \in \mathbb{R}^{N}$ one has $\lim _{|y| \rightarrow \infty} \Gamma(x, y)=0$.

For geometrical purposes it may occasionally be convenient to further require that:

$$
\lim _{y \rightarrow x} \Gamma(x, y)=\infty .
$$

This "blow-up" pole-condition is not part of our definition of a global fundamental solution, but will be required only if need be. A fundamental solution for $\mathscr{L}$, if it exists, is unique (a.e.): this follows from the Hörmander hypoellipticity theorem and from the Strong Maximum Principle. Following [35], we define the $\Gamma$-ball of centre $x$ and radius $r$ :

$$
\Omega_{r}(x):=\left\{y \in \mathbb{R}^{N}: \Gamma_{x}(y)>1 / r\right\} .
$$

Under the blow-up pole-condition, $\left\{\Omega_{r}(x)\right\}_{r}$ forms a basis of neighborhoods (as $r \downarrow 0)$, invading $\mathbb{R}^{N}$ (as $\left.r \uparrow \infty\right)$.

The Divergence Theorem and the $V$-divergence form of $\mathscr{L}$ imply the following surface mean-integral formula: whenever $u$ is $C^{2}$ on $\overline{\Omega(x, r)}$ it holds that 


$$
u(x)=m_{r}(u)(x)-\int_{\Omega_{r}(x)}\left(\Gamma_{x}(y)-\frac{1}{r}\right) \mathscr{L} u(y) \mathrm{d} \mu(y),
$$

where

$$
m_{r}(u)(x):=\int_{\partial \Omega_{r}(x)} u \mathscr{K}_{x} V \mathrm{~d} H^{N-1},
$$

with the kernel $\mathscr{K}_{x}$ being defined on $\mathbb{R}^{N} \backslash\{x\}$ by

$$
\mathscr{K}_{x}:=\frac{\left\langle A \nabla \Gamma_{x}, \nabla \Gamma_{x}\right\rangle}{\left|\nabla \Gamma_{x}\right|} .
$$

Unlike the classic Laplacian case, the kernel $\mathscr{K}_{x}(y)$ may vanish: this is indeed the case with the first Heisenberg group, where the associated $y \mapsto \mathscr{K}_{x}(y)$ vanishes precisely on the line $\left\{y_{1}=x_{1}, y_{2}=x_{2}\right\}$.

In general, since $\mathscr{K}_{x}(y)=0$ if and only if $\left\langle A(y) \nabla \Gamma_{x}(y), \nabla \Gamma_{x}(y)\right\rangle=0$, and the latter holds true if and only if $A(y) \nabla \Gamma_{x}(y)=0$, we see that $\mathscr{K}_{x}(y)=0$ precisely when

$$
A_{1} \Gamma_{x}(y)=\cdots=A_{N} \Gamma_{x}(y)=0,
$$

with the notation in (3). Thus, by Amano's controllability result [4], we infer that

$$
\mathscr{K}_{x}(y)>0 \text { on an open dense set in } \mathbb{R}^{N} \text {. }
$$

Arguing as in [1], this last fact can lead to an alternative proof of the Strong Maximum Principle for an $\mathscr{L}$ endowed with a global fundamental solution. Indeed, if $\mathscr{L} u \geq 0$ and if $x_{0} \in \Omega$ is a maximum point of $u$, we have

$$
u\left(x_{0}\right) \leq m_{r}(u)\left(x_{0}\right)=\int_{\partial \Omega_{r}\left(x_{0}\right)} u \mathscr{K}_{x_{0}} \mathrm{~d} H^{N-1} \leq u\left(x_{0}\right) .
$$

This immediately gives

$$
0=\int_{\partial \Omega_{r}\left(x_{0}\right)}\left(u\left(x_{0}\right)-u\right) \mathscr{K}_{x_{0}} \mathrm{~d} H^{N-1}
$$

if we integrate with respect to $r \in[0, R]$ and we use co-area formula, we get

$$
0=\int_{\Omega_{R}\left(x_{0}\right)}\left(u\left(x_{0}\right)-u\right)\left\langle A \nabla \Gamma_{x_{0}}, \nabla \Gamma_{x_{0}}\right\rangle \mathrm{d} \mu .
$$

Since $u\left(x_{0}\right)-u \geq 0$ and $\left\langle A \nabla \Gamma_{x_{0}}, \nabla \Gamma_{x_{0}}\right\rangle>0$ in an open dense set, we see that $u$ is constantly equal to $u\left(x_{0}\right)$ in $\Omega_{R}\left(x_{0}\right)$, so that $u$ is constant throughout $\Omega$ (which is connected).

The applications of mean-value formulas to Potential Theory are countless; among them we mention the applications to:

- Maximum Principles, [1];

- Gauss\&Koebe-type Theorems (characterization of the $\mathscr{L}$-harmonicity), [7]; 
- Montel-type Theorems (concerning the topology of the $\mathscr{L}$-harmonic functions), [7];

- many possible different characterizations of the $\mathscr{L}$-subharmonicity, [24, 25].

When sub-Laplacians on Carnot groups are involved, we also have applications to:

- Harnack inequalities and Liouville-type theorems, [21];

- Maximum Principles on unbounded domains, [22];

- Bôcher-type Theorems (on the removability of singularities), [23].

The vast applicability of mean-value formulas (deriving from the sole existence of $\Gamma$ ) makes it (very!) desirable to enlarge the class of PDOs for which the existence of a global fundamental solution (with the above well-behaved properties) is known. See Section 5.

\section{An invariant Harnack inequality}

The aim of this section is to describe how we could obtain an invariant and nonhomogeneous Harnack inequality for PDOs $\mathscr{L}$ of the form (1) but under different assumptions than those in Section 2. For instance, we drop our previous hypoellipticity assumptions $(\mathrm{HY})$ and $(\mathrm{HY})_{\varepsilon}$, in favor of more geometrical hypotheses. We shall skip any detail here, referring the interested reader to [8].

As we already discussed, a convenient framework to prove an invariant Harnack inequality for (1) is that of doubling metric spaces; hence, we suppose that the quadratic form of $\mathscr{L}$ can be controlled by a family of locally-Lipschitz vector fields $X=\left\{X_{1}, \ldots, X_{m}\right\}$ on $\mathbb{R}^{N}$, in the sense the $X$-elliptic operators introduced by Kogoj and Lanconelli in [60]: this means that there exist two constants $\lambda, \Lambda>0$ such that

$$
\lambda \sum_{j=1}^{m}\left\langle X_{j}(x), \xi\right\rangle^{2} \leq \sum_{i, j=1}^{N} a_{i, j}(x) \xi_{i} \xi_{j} \leq \Lambda \sum_{j=1}^{m}\left\langle X_{j}(x), \xi\right\rangle^{2}, \quad \forall x, \xi \in \mathbb{R}^{N} .
$$

Moreover, we assume that, with the associated Carnot-Carathéodory metric $d, N$-dimensional Euclidean space is endowed by $d$ with the structure of a doubling space (globally) and a Poincaré inequality holds true on any $d$-ball in space (this can be seen as another 'global' assumption). Here the doubling measure is again the measure $\mu$ introduced in (2), naturally associated with $\mathscr{L}$. These assumptions read as follows:

- assumption (D): there exists $Q>0$ such that

$$
\mu(B(x, 2 r)) \leq 2^{Q} \mu(B(x, r)), \quad \text { for every } x \in \mathbb{R}^{N} \text { and every } r>0 ;
$$

- assumption (P): there exists a constant $C_{P}>0$ such that, for every $x \in \mathbb{R}^{N}, r>0$ and every $u$ which is $C^{1}$ in a neighborhood of $B(x, 2 r)$ one has 


$$
f_{B(x, r)}\left|u(y)-u_{B(x, r)}\right| \mathrm{d} \mu(y) \leq C_{P} r f_{B(x, 2 r)}|X u(y)| \mathrm{d} \mu(y) .
$$

Here we used the following notations: $|X u|:=\sqrt{\sum_{j=1}^{m}\left|X_{j} u\right|^{2}}$ and

$$
f_{B(x, r)}\{\cdots\} \mathrm{d} \mu=\frac{1}{\mu(B(x, r))} \int_{B(x, r)}\{\cdots\} \mathrm{d} \mu, \quad u_{B(x, r)}:=f_{B(x, r)} u(y) \mathrm{d} \mu(y) .
$$

Under these assumptions (plus some further mild technical hypotheses on the $d$ topology ${ }^{1}$ ), one can derive Poincaré-Sobolev-type inequalities on the $d$-balls, following the ideas by Hajłasz and Koskela in [55].

Since we are interested in relating the global doubling condition and the global Poincaré inequality to the theory of subelliptic operators, it is natural to work in the setting of the Sobolev spaces associated with the family of vector fields $X$ with respect to the doubling measure $\mu$. Accordingly, we take for granted the associated notion of weak solution to $-\mathscr{L} u=g$ (when $g$ is in $L^{p}$ ).

Thanks to a density result of Meyers-Serrin type (see $[44,47]$ ), one can consider the Sobolev space $W^{1,2}(\Omega, X, \mu)$ as the closure of the space of the smooth functions in the norm of $W^{1,2}$, similarly to the classic case. This result, together with some Sobolev-type inequality on the $d$-balls (see also [20, 46, 50, 55]), is an important tool in order to obtain an adaptation of the well-established Moser iterative technique; as is well known, this powerful technique allowed us to obtain the following invariant and non-homogeneous Harnack inequality for $\mathscr{L}$ (with the consequent inner and boundary Hölder estimates):

Theorem 3 (Non-homogeneous, invariant Harnack inequality). Let the assumptions $(D)$ and $(P)$ be satisfied for $\mathscr{L}$ and for the doubling metric space $\left(\mathbb{R}^{N}, d, \mu\right)$. Let $\Omega \subseteq \mathbb{R}^{N}$ be an open set, and let $g \in L^{p}(\Omega)$, with $p>Q / 2$.

Then there exists a structural constant $C>0$ (only depending on the doubling/Poincaré constants $Q, C_{P}$, on the $X$-ellipticity constants $\lambda, \Lambda$ and on $p$ ) such that, for every $d$-ball $B(x, R)$ satisfying $\overline{B(x, 4 R)} \subset \Omega$, one has

$$
\sup _{B(x, R)} u \leq C\left(\inf _{B(x, R)} u+R^{2}\|g\|_{L^{p}(B(x, 4 R))}^{*}\right),
$$

for any nonnegative $W_{\text {loc }}^{1}$-weak solution $u$ of $-\mathscr{L} u=g$ in $\Omega$.

Here we used the notation

$$
\|g\|_{L^{p}(A)}^{*}:=\left(f_{A}|g|^{p} \mathrm{~d} \mu\right)^{1 / p}
$$

In the particular case when $g \equiv 0$, from Theorem 3 one obtains the homogeneous, invariant Harnack inequalities obtained by Kogoj and Lanconelli in [60, 61]. Again

\footnotetext{
${ }^{1}$ We assume that the metric space $\left(\mathbb{R}^{N}, d\right)$ is complete and its topology coincides with the Euclidean one.
} 
in the homogeneous case $g \equiv 0$, an invariant Harnack inequality under local doubling/Poincaré has been proved by Gutiérrez and Lanconelli in [53], for balls of small radii. In the same paper [53], the authors obtain a non-homogeneous invariant Harnack inequality, under the presence of some dilation-invariance property on the vector fields $X$ involved.

The summand $R^{2}\|g\|_{L^{p}(B(x, 4 R))}^{*}$ is bounded from above by

$$
\frac{R^{2}}{\mu(B(x, R))^{1 / p}}\|g\|_{L^{p}(\Omega)} ;
$$

when $R$ is small and $x$ lies in a compact set $K \subset \Omega$, there exists a constant $C(Q, K)>$ 0 such that (due to the doubling inequality (D)) the latter does not exceed

$$
C(Q, K) R^{2-Q / p}\|g\|_{L^{p}(\Omega)}
$$

Thus, our inequality (6) contains the analogous non-homogeneous, invariant Harnack inequality by Uguzzoni in [72], where it is considered the particular case when $x$ is confined in some compact set $K \subset \Omega$ and $R$ is very small. Roughly put, these more restrictive requirements on the $d$-balls involved are the drawback of the local doubling/Poincaré assumptions made in [72]; in the same spirit, our global inequality (6) (in the sense that it holds for any $d$-ball) is the product of our more onerous assumptions that doubling and Poincaré inequalities hold globally for any $d$-ball.

In a future investigation, we intend to apply Theorem 3 in order to prove the existence of a global fundamental solution for our $X$-elliptic operators $\mathscr{L}$ : this will require a deep insight of the Green function related to $\mathscr{L}$, plus the maximum principles by Gutiérrez and Lanconelli in [53].

\section{The existence of the fundamental solution for a class of PDOs}

In order to explain the main idea behind our technique for obtaining a fundamental solution for a (selected) class od PDOs, we describe an example. Let $\Delta_{n}=\sum_{j=1}^{n}\left(\partial_{x_{j}}\right)^{2}$ be the classic Laplace operator on $\mathbb{R}^{n}$; then we may say that $\Delta_{n+p}$ is a 'lifting' of $\Delta_{n}$ (for every $p \geq 1$ ) in the sense that

$$
\Delta_{n+p}(f \circ \pi)=\left(\Delta_{n} f\right) \circ \pi \quad \text { for every } f \in C^{\infty}\left(\mathbb{R}^{n}, \mathbb{R}\right),
$$

where $\pi: \mathbb{R}^{n+p} \rightarrow \mathbb{R}^{n}$ is the projection of $\mathbb{R}^{n+p}$ onto $\mathbb{R}^{n}$. If $n>2$, the fundamental solution of $\Delta_{n}$ (a constant multiple of $\|x\|^{2-n}$ ) can be recovered by that of $\Delta_{n+p}$ by a process that we may call 'saturation', in the sense that we integrate over the remaining $p$ coordinates: this comes from the integral identity ${ }^{2}$

\footnotetext{
${ }^{2}$ The identity follows from the change of variable $t=\|x\| \tau$ (with $\tau \in \mathbb{R}^{p}$ ).
} 


$$
c\left(\sqrt{x_{1}^{2}+\cdots+x_{n}^{2}}\right)^{2-n}=\int_{\mathbb{R}^{p}}\left(\sqrt{x_{1}^{2}+\cdots+x_{n}^{2}+t_{1}^{2}+\cdots+t_{p}^{2}}\right)^{2-n-p} \mathrm{~d} t_{1} \ldots \mathrm{d} t_{p},
$$

holding true for some constant $c$, whenever $n>2$. Incidentally, the above integral is convergent precisely when $n>2$. We aim to obtain an analogous process of lifting/saturation for other classes of PDOs.

To this end, we consider a set $\mathscr{X}=\left\{X_{1}, \ldots, X_{m}\right\}$ of linearly independent ${ }^{3}$ smooth vector fields on $\mathbb{R}^{n}$ satisfying the following assumptions:

(H1) $X_{1}, \ldots, X_{m}$ are homogeneous of degree 1 with respect to the non-isotropic dilations

$$
\delta_{\lambda}(x)=\left(\lambda^{\sigma_{1}} x_{1}, \ldots, \lambda^{\sigma_{n}} x_{n}\right),
$$

where $1=\sigma_{1} \leq \ldots \leq \sigma_{n}$ and $q:=\sum_{j=1}^{n} \sigma_{j}>2$ (the so-called homogeneous dimension related to $\delta_{\lambda}$ ); this means that (for any $i=1, \ldots, m$ )

$$
X_{i}\left(f \circ \delta_{\lambda}\right)=\lambda\left(X_{i} f\right) \circ \delta_{\lambda} \quad \forall \lambda>0, \quad \forall f \in C^{\infty}\left(\mathbb{R}^{n}, \mathbb{R}\right) .
$$

(H2) $X_{1}, \ldots, X_{m}$ satisfy the Hörmander rank condition:

$$
\operatorname{dim}\left\{X(x): X \in \operatorname{Lie}\left\{X_{1}, \ldots, X_{m}\right\}\right\}=n, \quad \text { for every } x \in \mathbb{R}^{n} .
$$

We then introduce the operator $\mathscr{L}$ naturally associated with $\mathscr{X}$, that is,

$$
\mathscr{L}=\sum_{j=1}^{m} X_{j}^{2} .
$$

Example 2. Let us consider, on Euclidean space $\mathbb{R}^{2}$, the Grushin vector fields:

$$
X_{1}=\partial_{x_{1}}, \quad X_{2}=x_{1} \partial_{x_{2}}
$$

Clearly, $X_{1}, X_{2}$ are independent (as differential operators ${ }^{4}$, even if not point-wise independent) and smooth; they are homogeneous of degree 1 with respect to $\delta_{\lambda}(x)=$ $\left(\lambda x_{1}, \lambda^{2} x_{2}\right)$; they satisfy the Hörmander rank condition. A lifting of $\mathscr{G}:=X_{1}^{2}+X_{2}^{2}$ is given by the PDO on $\mathbb{R}^{3}$ defined by

$$
\widetilde{\mathscr{G}}:=\left(\partial_{x_{1}}\right)^{2}+\left(\partial_{x_{3}}+x_{1} \partial_{x_{2}}\right)^{2}
$$

The idea of obtaining a fundamental solution for the Grushin operator $\mathscr{G}$ via a saturation argument applied to the (explicit!) fundamental solution of $\widetilde{\mathscr{G}}$ has already been exploited in the literature (e.g., in Bauer, Furutani, Iwasaki [9]; see also Calin,

\footnotetext{
${ }^{3}$ Throughout, we consider the set of the smooth vector fields on $\mathbb{R}^{N}$ as a real vector space and not as a $C^{\infty}$-module; therefore, the vector fields $X_{1}, \ldots, X_{m}$ are linearly dependent iff there exist $c_{1}, \ldots, c_{m} \in \mathbb{R}$, not all vanishing, such that $c_{1} X_{1}+\cdots+c_{m} X_{m}=0$ as a first order linear PDO (i.e., all of its coefficient functions with respect to the usual coordinate partial derivatives are identically equal to 0 ).

${ }^{4}$ This is not in contrast with the fact their coefficient vectors $(1,0)$ and $\left(0, x_{1}\right)$ are linearly dependent at $x_{1}=0$; see the previous footnote.
} 
Chang, Furutani, Iwasaki [31]; for more general PDOs on 2-step Carnot groups, see Beals, Gaveau, Greiner, Kannai [14]). Other references related to the (difficult) problem of obtaining explicit/integrally-represented fundamental solutions are the following ones: [2, 10,11, 12, 13, 15, 18, 19, 28, 32, 56]. See [16] for a wider list.

We intend to show that a process of lifting/saturation can always be performed for our PDO $\mathscr{L}$ in (7), when (H1) and (H2) are satisfied. Indeed, $\mathscr{L}$ admits a lifting which is a sub-Laplacian $\mathscr{L}_{\mathbb{G}}$ on some Carnot group $\mathbb{G}$; most importantly, since $\mathscr{L}_{\mathbb{G}}$ admits a global fundamental solution $\Gamma_{\mathbb{G}}$ (see Folland [42]), we shall show that a global fundamental solution $\Gamma$ for $\mathscr{L}$ can always be obtained by a saturation argument from $\Gamma_{\mathbb{G}}$. This gives the existence of $\Gamma$, together with an integral representation for it.

We describe more closely our procedure. The lifting method introduced by Rothschild and Stein [69] (which is, roughly put, a local fact) does not seem to be applicable. What is suited to our case is the global lifting for homogeneous operators proved by Folland in [43], which we now describe. We set $\mathfrak{a}:=\operatorname{Lie}\left\{X_{1}, \ldots, X_{m}\right\}$. Then $\mathfrak{a}$ is nilpotent and stratified:

$$
\mathfrak{a}=\mathfrak{a}_{1} \oplus \mathfrak{a}_{2} \oplus \cdots \oplus \mathfrak{a}_{r}
$$

with $\left[\mathfrak{a}_{1}, \mathfrak{a}_{i-1}\right]=\mathfrak{a}_{i}$ if $2 \leq i \leq r=\sigma_{n}$, and $\left[\mathfrak{a}_{1}, \mathfrak{a}_{r}\right]=\{0\}$, where we have also set $\mathfrak{a}_{1}:=\operatorname{span}\left\{X_{1}, \ldots, X_{m}\right\}$. We equip $\mathfrak{a}$ with the Campbell-Hausdorff operation

$$
X \diamond Y=X+Y+\frac{1}{2}[X, Y]+\frac{1}{12}[X,[X, Y]]-\frac{1}{12}[Y,[X, Y]]+\cdots,
$$

and with the family of dilations

$$
\Delta_{\lambda}(X)=\sum_{k=1}^{r} \lambda^{k} V_{k}, \quad \text { where } X=V_{1}+\cdots+V_{r} \text { and } V_{k} \in \mathfrak{a}_{k} .
$$

Folland's theorem ensures that the triple $\mathbb{A}:=\left(\mathfrak{a}, \diamond, \Delta_{\lambda}\right)$ is a homogeneous Carnot group, in the sense of [26]. Moreover, setting ${ }^{5}$

$$
\pi: \mathbb{A} \rightarrow \mathbb{R}^{n}, \quad \pi(X):=\exp (X)(0),
$$

the following properties hold true:

- $\pi$ is surjective;

- there exists a system $\left\{J_{1}, \ldots, J_{m}\right\}$ of Lie-generators of $\operatorname{Lie}(\mathbb{A})$ such that

$$
\mathrm{d}_{a} \pi\left(J_{i}\right)=\left(X_{i}\right)_{\pi(a)}, \quad a \in \mathbb{A} \quad \text { and } \quad i=1, \ldots, m .
$$

${ }^{5}$ Here $\exp (t X)(x)$ denotes the solution $t \mapsto \gamma(t)$ of the Cauchy problem

$$
\dot{\gamma}(t)=X(\gamma(t)), \quad \gamma(0)=x .
$$


In [16] we proved that something more can be done: we perform a suitable change of variable $T: \mathbb{R}^{N} \equiv \mathbb{A} \rightarrow \mathbb{R}^{N}$ turning $\pi$ into the canonical projection $\pi: \mathbb{R}^{n} \times \mathbb{R}^{p} \rightarrow \mathbb{R}^{n}$,

$$
\pi\left(x_{1}, \ldots, x_{n}, \xi_{1}, \ldots, \xi_{p}\right)=\left(x_{1}, \ldots, x_{n}\right),
$$

where $p=N-n=\operatorname{dim}\left(\operatorname{Lie}\left\{X_{1}, \ldots, X_{m}\right\}\right)-n$; this gives an explicit knowledge of the fibers of $\pi$ (all equal to $\mathbb{R}^{p}$ ).

Putting together Folland's lifting and our change of variable, we infer that there exists a homogeneous Carnot group $\mathbb{G}=\left(\mathbb{R}^{N}, \star, D_{\lambda}^{\star}\right)$ and there exists a system $\left\{Z_{1}, \ldots, Z_{m}\right\}$ of generators of $\operatorname{Lie}(\mathbb{G})$ such that

$$
Z_{i}=X_{i}+\sum_{j=1}^{p} r_{i, j}(x, \xi) \frac{\partial}{\partial \xi_{j}}, \quad \text { for every } i=1, \ldots, m .
$$

We also have

$$
D_{\lambda}^{\star}(x, \xi)=\left(\delta_{\lambda}(x), \delta_{\lambda}^{*}(\xi)\right)=\left(\delta_{\lambda}(x), \lambda^{s_{1}} \xi_{1}, \ldots, \lambda^{s_{p}} \xi_{p}\right) .
$$

Due to the mentioned result by Folland, the sub-Laplacian $\mathscr{L}_{\mathbb{G}}:=\sum_{j=1}^{m} Z_{j}^{2}$ has a unique global fundamental solution $\Gamma$ of the form

$$
\Gamma_{\mathbb{G}}((x, \xi),(y, \eta))=d^{2-q-\widetilde{q}}\left((x, \xi)^{-1} \star(y, \eta)\right), \quad(x, \xi) \neq(y, \eta),
$$

where $d$ is a suitable homogeneous norm on $\mathbb{G}$ and $\widetilde{q}=\sum_{j=1}^{p} s_{j}$ is the homogeneous dimension of $\mathbb{R}^{p}$ with respect to $\delta_{\lambda}^{*}$.

Now we can perform the announced saturation argument: it can be proved that the following is a fundamental solution for $\mathscr{L}$ :

$$
\Gamma(x, y):=\int_{\mathbb{R}^{p}} d^{2-q-\widetilde{q}}\left((x, 0)^{-1} \star(y, \eta)\right) \mathrm{d} \eta \quad(x \neq y) .
$$

To prove this, the main issue is to show that, for every fixed $x, y \in \mathbb{R}^{n}$ with $x \neq y$, one has

$$
(\mathrm{S}): \quad \mathbb{R}^{p} \ni \eta \mapsto d^{2-q-\widetilde{q}}\left((x, 0)^{-1} \star(y, \eta)\right) \text { is in } L^{1}\left(\mathbb{R}^{p}\right) .
$$

Some key facts for the proof of property $(\mathrm{S})$ are the following:

- for every $x, y \in \mathbb{R}^{n}$ with $x \neq y$, we have

$$
\Gamma_{\mathbb{G}}=d^{2-q-\widetilde{q}}\left((x, 0)^{-1} \star(y, \eta)\right) \in L_{\mathrm{loc}}^{1}\left(\mathbb{R}^{p}\right) ;
$$

- there exists a real $\mathbf{c}>0$ such that

$$
d^{2-q-\widehat{q}}(z, \zeta) \leq \mathbf{c}\left(\sum_{i=1}^{p}\left|\zeta_{i}\right|^{1 / s_{i}}\right)^{2-q-\widetilde{q}}=: \varphi(\zeta), \quad \text { for all }(z, \zeta) \in \mathbb{R}^{N}
$$

- via a suitable change of variable, $\Gamma_{\mathbb{G}}\left((x, 0)^{-1} \star(y, \eta)\right)$ is $\eta$-integrable at infinity if the same is true of $\varphi$; 
- $\varphi$ is integrable at infinity (by a homogeneous argument on annuli).

Once (S) is established, it is not too difficult to recognize that $\Gamma$ is a fundamental solution for $\mathscr{L}$, further satisfying the following properties:

1. setting $\mathscr{O}:=\left\{(x, y) \in \mathbb{R}^{n} \times \mathbb{R}^{n}: x \neq y\right\}$, then $\Gamma \in C^{\infty}(\mathscr{O}, \mathbb{R})$;

2. $\Gamma(x, y)>0$ on $\mathscr{O}$;

3. $\Gamma(x, y)=\Gamma(y, x)$ for every $x \neq y$;

4. $\Gamma \in L_{\text {loc }}^{1}\left(\mathbb{R}^{n} \times \mathbb{R}^{n}\right)$;

5. $\Gamma\left(\delta_{\lambda}(x), \delta_{\lambda}(y)\right)=\lambda^{2-q} \Gamma(x, y)$.

In the forthcoming paper [17], we shall consider the parabolic case as well. More precisely, we consider once again a set a set $\mathscr{X}=\left\{X_{1}, \ldots, X_{m}\right\}$ of linearly independent smooth vector fields on $\mathbb{R}^{n}$ satisfying the above assumptions (H1) and (H2). We define

$$
\mathscr{H}:=\mathscr{L}-\partial_{t}=\sum_{j=1}^{m} X_{j}^{2}-\partial_{t}, \quad \text { on } \mathbb{R}^{1+n}=\mathbb{R}_{t} \times \mathbb{R}_{x}^{n} .
$$

We prove the existence of a "well-behaved" global fundamental solution (usually referred to as a heat kernel) for the parabolic PDO $\mathscr{H}$. A lifting/saturation approach can be performed. Indeed, let $Z_{1}, \ldots, Z_{m}$ be as above; then the operator

$$
\mathscr{H}_{\mathbb{G}}:=\mathscr{L}_{\mathbb{G}}-\partial_{t}=\sum_{j=1}^{m} Z_{j}^{2}-\partial_{t}, \quad \text { on } \mathbb{R}^{1+N}=\mathbb{R}_{t} \times \mathbb{R}_{(x, \xi)}^{N}
$$

is a lifting of $\mathscr{H}$ on $\mathbb{R} \times \mathbb{G} \equiv \mathbb{R}^{1+N}$. As regards the operator $\mathscr{H}_{\mathbb{G}}$, it is well-know that there exists a smooth map $\gamma_{\mathbb{G}}: \mathbb{R}^{1+N} \backslash\{0\} \rightarrow \mathbb{R}$ such that the function

$$
\Gamma_{\mathbb{G}}(t, x, \xi ; s, y, \eta):=\gamma_{\mathbb{G}}\left(\left(s-t,(x, \xi)^{-1} \star(y, \eta)\right), \quad(t, x, \xi) \neq(s, y, \eta)\right.
$$

is a global fundamental solution for $\mathscr{H}_{\mathbb{G}}$ (here, $\star$ is the composition law of $\mathbb{G}$ ). Since $\mathscr{H}_{\mathbb{G}}$ is a lifting for $\mathscr{H}$, it is natural to define

$$
\Gamma(t, x ; s, y):=\int_{\mathbb{R}^{p}} \gamma_{\mathbb{G}}\left(s-t,(x, 0)^{-1} \star(y, \eta)\right) \mathrm{d} \eta .
$$

The main issue here is to prove that, for every fixed $(t, x) \neq(s, y) \in \mathbb{R}^{1+n}$, one has

$$
(\mathrm{S})^{\prime}: \quad \mathbb{R}^{p} \ni \eta \mapsto \gamma_{\mathbb{G}}\left(s-t,(x, 0)^{-1} \star(y, \eta)\right) \text { is in } L^{1}\left(\mathbb{R}^{p}\right) .
$$

Key tools for proving property (S)' are:

- the existence of a constant $\mathbf{c}>0$ such that, for every $(x, \xi) \in \mathbb{R}^{N}$ and every $t>0$, the following uniform Gaussian estimates hold true:

$$
\frac{1}{\mathbf{c} t^{Q / 2}} \exp \left(-\frac{\mathbf{c} d^{2}(x, \xi)}{t}\right) \leq \gamma_{\mathbb{G}}(t, x, \xi) \leq \frac{\mathbf{c}}{t^{Q / 2}} \exp \left(-\frac{d^{2}(x, \xi)}{\mathbf{c} t}\right)
$$

here, $d$ is the same homogeneous norm introduced in the stationary case; 
- the estimates for $d$ exploited in the stationary case.

Once (S)' is established, one can show that the function $\Gamma$ is well-defined and is a global fundamental solution for $\mathscr{H}$, further satisfying the following properties:

1. $\Gamma \geq 0$ and $\Gamma(t, x ; s, y)=0$ if and only if $s \leq t$;

2. $\Gamma$ depends on $t$ and $s$ only through the difference $s-t$; moreover, $\Gamma$ is symmetric with respect to the space variables;

3. $\Gamma\left(\lambda^{2} t, \delta_{\lambda}(x) ; \lambda^{2} s, \delta_{\lambda}(y)\right)=\lambda^{-q} \Gamma(t, x ; s, y)$;

4. $\Gamma$ is smooth out of the diagonal of $\mathbb{R}^{1+n} \times \mathbb{R}^{1+n}$;

5. $\Gamma \in L_{\text {loc }}^{1}\left(\mathbb{R}^{1+n} \times \mathbb{R}^{1+n}\right)$;

6. for every fixed $(t, x) \in \mathbb{R}^{1+n}$ we have

$$
\int_{\mathbb{R}^{n}} \Gamma(t, x ; s, y) \mathrm{d} y=1, \quad \text { for every } s>t ;
$$

Acknowledgements. The results of this paper were presented by the author at the Conference "Noncommutative Analysis and Partial Differential Equations", 11-15 April, 2016, Imperial College, London; the author wishes to express his gratitude to the Organizing Committee of the Conference for the hospitality.

\section{References}

1. Abbondanza, B., Bonfiglioli, A.: On the Dirichlet problem and the inverse mean value theorem for a class of divergence form operators, J. London Math. Soc., 1-26 (2012); doi: $10.1112 / \mathrm{jlms} / \mathrm{jds} 050$.

2. A. Agrachev, U. Boscain, J.-P. Gauthier, F. Rossi: The intrinsic hypoelliptic Laplacian and its heat kernel on unimodular Lie groups, J. Funct. Anal. 256 (2009), 2621-2655.

3. Aimar, H., Forzani, L., Toledano, R.: Hölder regularity of solutions of PDE's: a geometrical view, Comm. Partial Differential Equations 26 (2001), 1145-1173.

4. Amano, K.: A necessary condition for hypoellipticity of degenerate elliptic-parabolic operators, Tokyo J. Math. 2 (1979) 111-120.

5. Barlow, M.T., Bass, R.F.: Stability of parabolic Harnack inequalities, Trans. Amer. Math. Soc. 356 (2004), 1501-1533.

6. E. Battaglia, S. Biagi, A. Bonfiglioli: The strong maximum principle and the Harnack inequality for a class of hypoelliptic non-Hörmander operators, Ann. Inst. Fourier (Grenoble) 66 (2016), no. 2, 589-631.

7. Battaglia, E., Bonfiglioli, A.: Normal families of functions for subelliptic operators and the theorems of Montel and Koebe J. Math. Anal. Appl. 409 (2014), 1-12.

8. Battaglia, E., Bonfiglioli, A.: The Harnack inequality for a class of sub-elliptic operators under global doubling and Poincaré assumptions, preprint (2017).

9. W. Bauer, K. Furutani, C. Iwasaki: Fundamental solution of a higher step Grushin type operator, Adv. Math. 271 (2015), 188-234.

10. R. Beals: Exact fundamental solutions, "Équations aux Dérivées Partielles" (Saint-Jean-deMonts, 1998), Exp. No. I, 9 pp., Univ. Nantes, Nantes, 1998.

11. R. Beals, B. Gaveau, P. Greiner: On a geometric formula for the fundamental solution of subelliptic Laplacians, Math. Nachr. 181 (1996), 81-163.

12. R. Beals, B. Gaveau, P. Greiner: The Green function of model step two hypoelliptic operators and the analysis of certain tangential Cauchy Riemann complexes, Adv. Math. 121 (1996), no. 2, 288-345. 
13. R. Beals, B. Gaveau, P. Greiner, Y. Kannai: Exact fundamental solutions for a class of degenerate elliptic operators, Comm. Partial Differential Equations 24 (1999), no. 3-4, 719-742.

14. R. Beals, B. Gaveau, P. Greiner, Y. Kannai: Transversally elliptic operators, Bull. Sci. Math. 128 (2004), no. 7, 531-576.

15. R. Beals, P. Greiner, B. Gaveau,: fundamental solutions for some highly degenerate elliptic operators, J. Funct. Anal. 165 (1999), no. 2, 407-429.

16. S. Biagi, A. Bonfiglioli: The existence of a global fundamental solution for homogeneous Hörmander operators via a global Lifting method, Proc. London Math. Soc., 114 (2017), $855-889$.

17. S. Biagi, A. Bonfiglioli: Global heat kernels for homogeneous Hörmander operators and applications, in preparation (2017).

18. T. Bieske: Fundamental solutions to the p-Laplace equation in a class of Grushin vector fields, Electron. J. Differential Equations 2011 (2011), No. 84, 1-10.

19. T. Bieske, J. Gong: The p-Laplace equation on a class of Grushin-type spaces, Proc. Amer. Math. Soc. 134 (2006), 3585-3594.

20. M. Biroli, U. Mosco: Sobolev inequalities on homogeneous spaces. Potential theory and degenerate partial differential operators (Parma). Potential Anal. 4 (1995), 311-324.

21. A. Bonfiglioli, E. Lanconelli: Liouville-type theorems for real sub-Laplacians, Manuscripta Math. 105, 111-124 (2001).

22. A. Bonfiglioli, E. Lanconelli: Subharmonic functions on Carnot groups, Math. Ann. 325, 97-122 (2003).

23. A. Bonfiglioli, E. Lanconelli: Gauge functions, Eikonal equations and Bôcher's theorem on stratified Lie groups, Calc. Var. Partial Differential Equations 30, 277-291 (2007).

24. Bonfiglioli, A., Lanconelli, E.: Subharmonic functions in sub-Riemannian settings, J. Eur. Math. Soc. 15 (2013), 387-441.

25. Bonfiglioli, A., Lanconelli, E., Tommasoli, A.: Convexity of average operators for subsolutions to subelliptic equations Analysis \& PDE, 7 (2014), 345-373.

26. A. Bonfiglioli, E. Lanconelli, F. Uguzzoni: Stratified Lie groups and potential theory for their sub-Laplacians, Springer Monographs in Mathematics, Springer: Berlin, 2007.

27. J.-M. Bony: Principe du maximum, inégalité de Harnack et unicité du problème de Cauchy pour les opérateurs elliptiques dégénérés, Ann. Inst. Fourier (Grenoble), 19 (1969), $277-$ 304.

28. U. Boscain, J.-P. Gauthier, F. Rossi: The hypoelliptic heat kernel over three-step nilpotent Lie groups, Sovrem. Mat. Fundam. Napravl. 42 (2011), 48-61; transl. in: J. Math. Sci. (N. Y.) 199 (2014), no. 6, 614-628.

29. Bramanti, M., Brandolini, L., Lanconelli, E., Uguzzoni, F.: Non-divergence equations structured on Hörmander vector fields: heat kernels and Harnack inequalities, Mem. Amer. Math. Soc. 204 (2010), n.961.

30. Brelot, M.: Axiomatique des fonctions harmoniques, Séminaire de Mathématiques Supérieures, 14 (Été, 1965), Les Presses de l’Université de Montréal, Montréal, 1969.

31. O. Calin, D.-C. Chang, K. Furutani, C. Iwasaki: Heat kernels for elliptic and sub-elliptic operators, Methods and techniques. Applied and Numerical Harmonic Analysis. Birkhäuser/Springer, New York, 2011.

32. L. Capogna, D. Danielli, N. Garofalo: Capacitary estimates and subelliptic equations, Amer. J. Math. 118 (1996), 1153-1196.

33. Chanillo, S., Wheeden, R.L.: Harnack's inequality and mean-value inequalities for solutions of degenerate elliptic equations, Comm. Partial Differential Equations 11 (1986), 11111134.

34. Christ., M.: Hypoellipticity in the infinitely degenerate regime, in: Complex Analysis and Geometry (J.D. McNeal, ed.), Ohio State Univ. Math Res. Inst. Publ. 9, Walter de Gruyter, Berlin, 2001, pp. 59-84.

35. Citti, G., Garofalo, N., Lanconelli, E.: Harnack's inequality for sum of squares of vector fields plus a potential, Amer. J. Math. 115 (1993), 699-734.

36. Di Fazio, G., Gutiérrez, C.E., Lanconelli, E.: Covering theorems, inequalities on metric spaces and applications to PDE's, Math. Ann. 341 (2008), 255-291. 
37. Fabes, E.B., Jerison, D., Kenig, C.E.: The Wiener test for degenerate elliptic equations, Ann. Inst. Fourier (Grenoble) 32 (1982), 151-182.

38. Fabes, E.B., Kenig, C.E., Serapioni, R.P.: The local regularity of solutions of degenerate elliptic equations, Comm. Partial Differential Equations 7 (1982), 77-116.

39. Fefferman, C., Phong, D.H.: Subelliptic eigenvalue problems, Wadsworth Math. Ser. (Chicago, Ill., 1981), Wadsworth, Belmont, CA, 1983, pp. 590-606.

40. Fefferman, C., Phong, D.H.: The uncertainty principle and sharp Garding inequalities, Comm. Pure Appl. Math. 34 (1981), 285-331.

41. Fediŭ, V.S.: On a criterion for hypoellipticity, Math. USSR Sb. 14 (1971), 15-45.

42. G.B. Folland: Subelliptic estimates and function spaces on nilpotent Lie groups, Ark. MAt. 13 (1975), 161-207.

43. G.B. Folland: On the Rothschild-Stein lifting theorem, Comm. Partial Differential Equations 2 (1977), 161-207.

44. B. Franchi, P. Hajłasz, P. Koskela: Definitions of Sobolev classes on metric spaces, Ann. Inst. Fourier (Grenoble), 49 (1999), 1903-1924.

45. Franchi, B., Lanconelli, E.: Une condition géométrique pour l'inégalité de Harnack, J. Math. Pures Appl. 64 (1985), 237-256.

46. B. Franchi, G. Lu, R.L. Wheeden: A relationship between Poincaré-type inequalities and representation formulas in spaces of homogeneous type, Internat. Math. Res. Notices 1996, no. $1,1-14$.

47. B. Franchi, R. Serapioni, F. Serra Cassano: Approximation and imbedding theorems for weighted Sobolev spaces associated with Lipschitz continuous vector fields, Bollettino U.M.I B (7) 11 (1997), 83-117.

48. Garofalo, N., Lanconelli, E.: Asymptotic behavior of fundamental solutions and potential theory of parabolic operators with variable coefficients, Math. Ann. 283 (1989), 211-239.

49. Garofalo, N., Lanconelli, E.: Level sets of the fundamental solution and Harnack inequality for degenerate equations of Kolmogorov type, Trans. Amer. Math. Soc. 321 (1990), 775-792.

50. N. Garofalo, D.-M. Nhieu: Isoperimetric and Sobolev inequalities for Carnot-Carathéodory spaces and the existence of minimal surfaces, Comm. Pure Appl. Math., 49 (1996), 10811144.

51. Grigor'yan, A., Saloff-Coste, L.: Stability results for Harnack inequalities, Ann. Inst. Fourier (Grenoble) 55 (2005), 825-890.

52. Gutiérrez, C.E.: Harnack's inequality for degenerate Schrödinger operators, Trans. Amer. Math. Soc. 312 (1989), 403-419.

53. Gutiérrez, C.E., Lanconelli, E.: Maximum principle, nonhomogeneous Harnack inequality, and Liouville theorems for X-elliptic operators, Comm. Partial Differential Equations 28 (2003), 1833-1862.

54. Hebisch, W., Saloff-Coste, L.: On the relation between elliptic and parabolic Harnack inequalities, Ann. Inst. Fourier (Grenoble) 51 (2001), 1437-1481.

55. P. Hajłasz, P. Koskela: Sobolev met Poincaré, Mem. Amer. Math. Soc., 145 (2000).

56. J. Heinonen, I. Holopainen: Quasiregular maps on Carnot groups, J. Geom. Anal. 7 (1997), 109-148.

57. Indratno, S., Maldonado, D., Silwal, S.: On the axiomatic approach to Harnack's inequality in doubling quasi-metric spaces, J. Differential Equations 254 (2013), 3369-3394.

58. Jerison, D.S., Sánchez-Calle, A.: Subelliptic, second order differential operators, in: Complex Analysis III, Proc. Spec. Year, College Park 1985-86, Lect. Notes Math. 1277 (1987) 46-77.

59. Kinnunen, J., Marola, N., Miranda, M., Paronetto, F.: Harnack's inequality for parabolic De Giorgi classes in metric spaces, Adv. Differential Equations 17 (2012), 801-832.

60. A. E. Kogoj, E. Lanconelli: $X$-elliptic operators and $X$-elliptic distances, Contributions in honor of the memory of Ennio De Giorgi. Ricerche Mat. 49 (2000), 223-243.

61. A.E. Kogoj, E. Lanconelli: Liouville theorem for X-elliptic operators, Nonlinear Anal. 70 (2009), 2974-2985.

62. Kohn, J.J.: Hypoellipticity of some degenerate subelliptic operators, J. Funct. Anal. 159 (1998), 203-216. 
63. Kusuoka, S., Stroock, D.: Applications of the Malliavin calculus. II, J. Fac. Sci. Univ. Tokyo Sect. IA Math. 32 (1985), 1-76.

64. Mohammed, A.: Harnack's inequality for solutions of some degenerate elliptic equations, Rev. Mat. Iberoamericana 18 (2002), 325-354.

65. Morimoto, Y.: A criterion for hypoellipticity of second order differential operators, Osaka J. Math. 24 (1987), 651-675.

66. Parmeggiani, A.: A remark on the stability of $C^{\infty}$-hypoellipticity under lower-order perturbations, J. Pseudo-Differ. Oper. Appl. 6 (2015), 227-235.

67. Pascucci, A., Polidoro, S.: A Gaussian upper bound for the fundamental solutions of a class of ultraparabolic equations, J. Math. Anal. Appl. 282 (2003), 396-409.

68. Pascucci, A., Polidoro, S.: Harnack inequalities and Gaussian estimates for a class of hypoelliptic operators, Trans. Amer. Math. Soc. 358 (2006), 4873-4893

69. L.P. Rothschild, E.M. Stein: Hypoelliptic differential operators and nilpotent groups, Acta Math. 137 (1976), 247-320.

70. Saloff-Coste, L.: Parabolic Harnack inequality for divergence-form second-order differential operators, Potential Anal. 4 (1995), 429-467.

71. Stein, E.M.: An example on the Heisenberg group related to the Lewy operator, Invent. Math. 69 (1982), 209-216.

72. Uguzzoni, F.: Estimates of the Green function for X-elliptic operators, Math. Ann. 361 (2015), 169-190. 